\section{Current contrast during hypothalamic self-stimulation}

MARTIN J. GERBEN, U.S. Army Research Institute of Environmental Medicine, Natick, Mass. 01760

Hypothalamic self-stimulation rates of six rats were measured under varying combinations of the current intensities of the reinforcing and continuous background stimuli at the same hypothalamic locus. Response rate increased with intensity of reinforcement, up to a point and then remained constant. Reductions in response rate were associated with increasing background current. $A$ second experiment demonstrated that locomotor behavior increased with increasing background current during self-stimulation sessions. Results suggest that background stimulation elicited responses which were incompatible with operant responding.

There is considerable evidence that responses to particular environmental stimuli depend not only on the stimuli themselves, but also on the stimulus context in which they occur (Bevan, 1968). Much of this data has been interpreted in terms of perceptual theory (Helson, 1964; Wapner \& Werner, 1957) although Bevan (1966) has reviewed contextual effects on reinforcement of behavior of both human and subhuman species. Recently, MacDougall \& Bevan (1968) found that both peripheral and intracranial electric shock increased the rate of subsequent operant responding reinforced by subcortical brain stimulation.

The present experiments further investigated the effects of contextual brain stimulation on the rate of operant responses reinforced by brain stimulation. Specifically, the rate of hypothalamic self-stimulation was measured as a function of varying intensities of concurrent electrical background stimulation (EBS) of the same hypothalamic locus. Several theoretical positions would predict a decrease in response rate associated with increasing EBS. (1) Adaptation Hypothesis: Adaptation to prolonged EBS would be expected to decrease the effective magnitude of the reinforcing stimulus. Presumably, such a decrease would progress gradually as adaptation occurred. (2) Incompatibility Hypothesis: Behaviors elicited by EBS would interfere with operant responding. These elicited behaviors include eating, drinking, gnawing, grooming, manipulation and locomotion (Christopher \& Butter, 1968; Gerben, 1968; Komisaruk \& Olds, 1968; Rosenquist \& Hoebel, 1968). The decrease in response rate would be expected EBS. to occur relatively rapidly after the onse tof EBS due to the short latencies associated with the elicited behaviors. (3) Aversiveness Hypothesis: Long durations of stimulation of positive reinforcement loci apparently become aversive (e.g., Miller, 1958). This hypothesis implies that after prolonged EBS a rat would avoid any increase in aversive stimulation. Thus, it would be expected that self-stimulation would cease completely when relatively intense EBS was presented.

\section{EXPERIMENT 1}

Subjects

Six male Sprague-Dawley rats, $300-400 \mathrm{~g}$, were implanted with bipolar electrodes to the posterior hypothalamus $(3.25 \mathrm{~mm}$ posterior to the bregma, $0.75 \mathrm{~mm}$ lateral to the midline, and $9.00 \mathrm{~mm}$ beneath the surface of the skull). Ss were allowed 2 weeks' recovery after surgery and were free fed for the duration of the experiments. Placements were histologically verified subsequent to the running of the experiments.

\section{Apparatus}

Self-stimulation occurred in a $9 \times 9 \times 18$-in. Plexiglas operant conditioning chamber fitted with a Lehigh Valley Model 1352 rat lever. Solid-state program- ming equipment and a Cirass Model BPSI stimulatur were used. Each reinforcing stimulus consisted of a $0.2-\mathrm{sec}$ train of biphasic square wave pulse pairs $(0.1-\mathrm{msec}$ duration, $0.1-\mathrm{msec}$ interpulse interval, 100-pps repetition rate) delivered immediately after the operant response. EBS consisted of constant trains of similar stimulation and lasted from the offset of each reinforcing stimulus to the onset of the next. The intensity of stimulation was calculated using Ohm's law after observing the peak voltage across a $10 \mathrm{~K}$ resistor in series with $S$.

\section{Design and Procedure}

During the initial 10 sessions of the experiment (training), each lever deflection was reinforced at an intensity of $2.0 \mathrm{~mA}$. No background stimulation was presented and each training session was $1 \mathrm{~h}$ long. During the next five sessions (testing), both the intensity of the reinforcing stimulus and the intensity of the background stimulus were systematically varied as follows: (1) Five intensities of the reinforcing stimulus were used $(1.2,1.6,2.0,2.4$, and $2.8 \mathrm{~mA})$, each value on a different day, order randomized. (2) Four relative intensities of $\operatorname{EBS}(0,25$, 50 , and $75 \%$ of the reinforcing stimulus intensity) were factorially combined with the values of the reinforcing stimulus. Each testing session began with a 15-min "warm-up" period during which responding was initiated and maintained under the appropriate intensity of the reinforcing

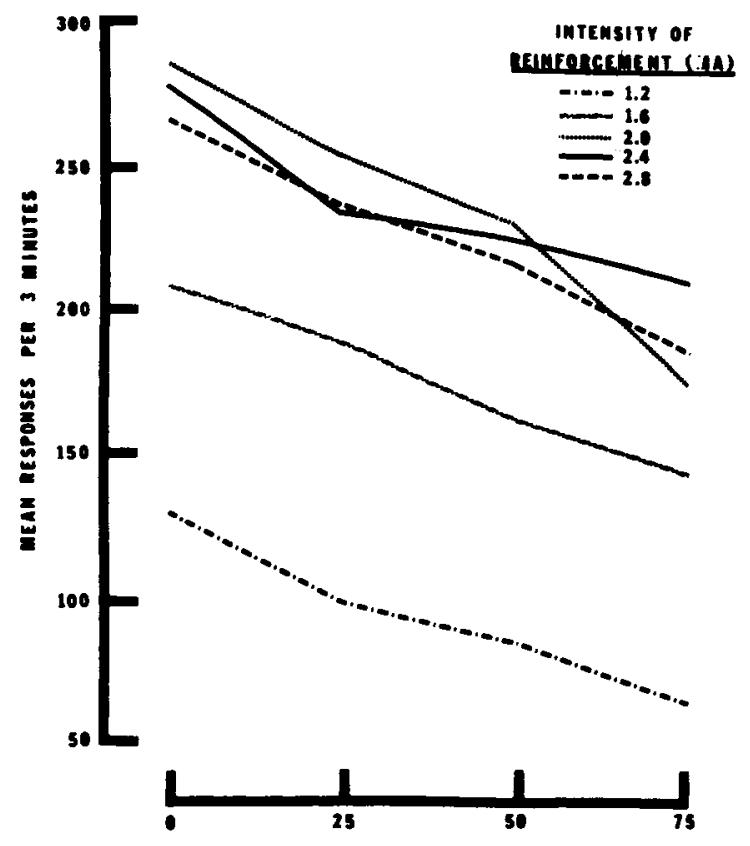

eacmenoum cunnent (PEncent of aeimfoncime stimulus)

Fig. 1. Mean responses per 3-min period as a function of the intensity of the reinforcement and the intensity of the 


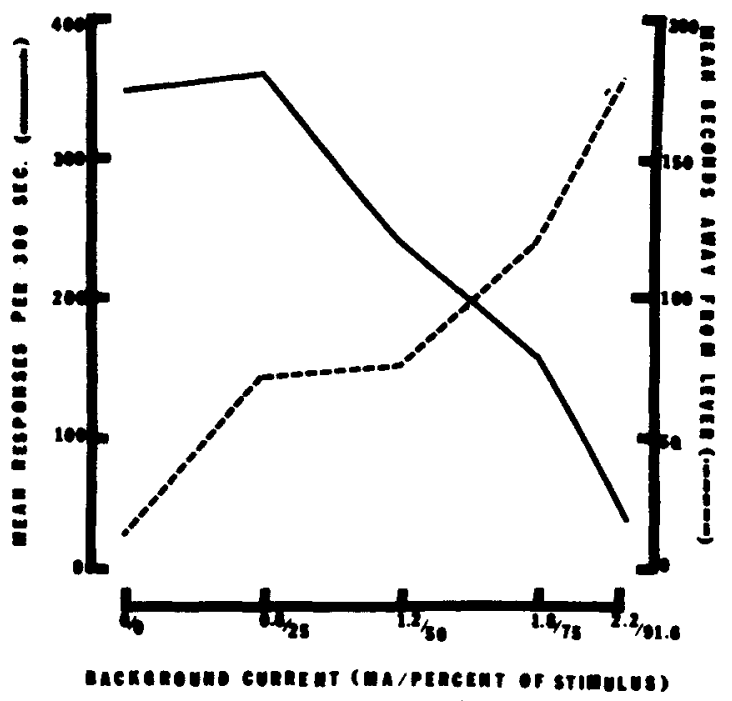

Fig. 2. Mean responses per 5-min $(300$-sec) period and mean time aw ay from the lever as functions of the EBS intensity for a 2.4-mA reinforcement intensity.

stimulus. During this period, no EBS was presented. The remainder of the session was divided into 15 consecutive 3 -min periods. Experimental periods alternated with recovery periods. During each of the odd numbered (experimental) periods, EBS assumed intensities of $0,25,50$, or $75 \%$ of the reinforcing stimulus, each intensity being presented twice, order randomized. During the even numbered (recovery) periods, the background stimulation intensity was zero. The number of responses per 3-min period was measured under each experimental condition. In addition, the number of responses per each 10 -sec interval within each 3-min period was measured in order to provide data concerning intraperiod sequential effects.

Results and Discussion

Figure 1 shows group mean number of responses per 3-min period as a function of the absolute intensity of the reinforcing stimulus and the relative intensity of the background stimulus. An analysis of variance indicated statistically significant $(p<.01)$ main effects of both variables. Response rate increased with increasing intensity of the reinforcement up to $2.0 \mathrm{~mA}$ $(F=7.61, d f=4 / 20)$ and decreased with increasing background current $(F=6.36$, $\mathrm{df}=3 / 15$ ). These results indicate: (1) EBS does not have to be reduced to zero between reinforcements in order to maintain some degree of self-stimulation. This result is contrary to what was predicted from the Aversiveness Hypothesis mentioned above. That is, if hypothalamic stimulation was already aversive due to prolonged EBS, why would $S s$ respond at all, especially when responding resulted in an increase in the intensity of stimulation that was already aversive? (2) The rate of hypothalamic self-stimulation is affected by the context of brain stimulation in which the reinforcing stimulus occurs. Inspection of Fig. 1 suggests that the absolute reduction in response rate is a direct linear function of the ratio of the intensities of EBS to the reinforcement. (3) Changes in response rate across $10-\mathrm{sec}$ intervals within each 3 -min period were not statistically significant. The latter result does not support the Adaptation Hypothesis but is consistent with the Incompatibility Hypothesis. Thus, the results of Experiment 1 suggest that EBS elicited behaviors which interfered with operant responding.

\section{EXPERIMENT 2}

The second experiment was a more direct test of the Incompatibility Hypothesis. Locomotor activity was measured concurrently with self-stimulation under several different EBS intensities. The same six Ss were trained to press a lever that was mounted on the wall of a Lehigh Valley Model 1497Q circular (24-in. diam) activity cage. The floor of this cage is divided into four quadrants. While lever pressing, the rat is always located on the lever-proximal quadrant. The amount of time spent on the remaining three quadrants was taken to be a measure of locomotor activity. During six experimental sessions, the temporal param. eters of reinforcement were identical to that used in Experiment 1 with the intensity remaining constant at $2.4 \mathrm{~mA}$. The first five sessions were 1 -h training sessions in which no EBS was presented. During the sixth session, five 5-min periods of background stimulation (assuming values of $0.0,0.6,1.2$,
1.8 , and $2.2 \mathrm{~mA}$ ) were alternated with $1-\mathrm{min}$ recovery periods during which no background stimulation occurred. The background stimulation intensity remained constant throughout each $5-\mathrm{min}$ period and the order of presentation of intensities across 5-min periods was randomized.

Results and Discussion

Figure 2 shows both the mean number of responses and the mean time spent off the lever-proximal quadrant per 5 -min period as a function of background stimulus intensity. Inspection of this figure indicates that locomotor activity increased as response rate decreased. These results support the Incompatibility Hypothesis. EBS elicited locomotor behavior which was incompatible with operant responding. Visual observation of $S s$ and cumulative records taken during this experiment indicate that the temporal distribution of operant and locomotor responses was quite regular over the middle range of EBS intensities. Typically, Ss would exhibit fairly uniform alternating periods of operant and locomotor responses. Under low EBS intensities, operant responding predominated while the opposite occurred under high background intensities. It should be noted that the results of Experiment 2 are partially correlational and no cause and effect relationship can be asserted between increase in locomotion and the decrease in operant responding. However, increases in locomotion with current intensity has been previously observed in both the running wheel (Gerben, 1968) ait the open field (Christopher \& Butter, 1968). Thus, taken as a whole, the results of the present two studies seem to favor the Incompatibility Hypothesis. That is, EBS elicited locomotor responses which were at least partially incompatible with operant responding.

\section{REFERENCES}

BEVAN, W. An adaptation-level interpretation of reinforcement. Perceptual \& Motor Skills, 1966 , 23, 511-531.

BEVAN, W. The contextual basis of behavior. American Psychologist, 1968, 23,701-714.

CHRISTOPHER, S., \& BUTTER, C. Consummatory behaviors and locomotor exploration evoked from self-stimulation sites in rats. Joumal of Comparative \& Physiological Psy chology, 1968,66, 335-339.

GERBEN, M. Running elicited by hypothalamic stimulation. Psychonomic Science, 1968, 12 , 19.20 .

HELSON, H. Adaptation-level theory. New York: Harper and Row, 1964.

KOMISARUK, B., \& OLDS, J. Neuronal comelates of behavior in freely moving rats. Science, 1968 , $161,810-812$.

MlLLFR, N. Central stimulation and other approaches to motivation and reward. American Psy chologist. 1958, 13, 100-108.

ROSFNQUIST, A., \& HOEBEL, B. Wheel running clicited by electrical stimulation of the brain. Physiology \& Behavior, 1968, 3, 563-566.

WAPNER, S., \& WERNER, H. Perceptual development. Worcester, Mass: Clark Unive rsity Press, 1957. 